\title{
Title: Stereoselective synthesis of highly-congested tetralin-fused spirooxindoles with hydroxyl group: net oxygen atom induced hydride shift/cyclization process
}

\author{
Authors: Dan Sakai, ${ }^{1}$ Mizuki Machida, ${ }^{1}$ and Keiji Mori ${ }^{1 *}$ \\ Affiliations: ' Department of Applied Chemistry, Graduate School of Engineering, Tokyo \\ University of Agriculture and Technology, 2-24-16 Nakacho, Koganei, Tokyo 184-8588 \\ *To whom correspondence should be addressed. \\ E-mail: k_mori@cc.tuat.ac.jp
}

\begin{abstract}
Highly stereoselective synthesis of tetralin-fused spirooxindoles with two contiguous stereogenic centers. In the present reaction, not only [1,5]-hydride shift/cyclization process, but also replacemnt of nitrogen atom to oxygen atom ocurred smoothly to give target structure with hydroxy grop in good chemical yields with good to excellent diastereoselectivities (up to $d . r .=>20: 1$ ). Investigation of the reaction mechanism suggested that this "atom-replacement" event ocurred via the iminium cation intermediates.
\end{abstract}

\section{Main text}

Hybrid molecule, which contains two or more number of pharmacore, is an important class of compound in the pharmaceutical and agrochemical fields. Because of the significant improvement of biological activity compared to the compound with single pharmacore and their structural complexity, much effort has been devoted to the development of synthetic method. ${ }^{1}$ Among the hybrid compounds, spirooxindoles having tetralin core is expected to be an useful organic molecules because each structure itself exhibits potent biological activities. $^{2-4}$ But surprisingly, the efficient method leading to this class of skeleton was quite limited..$^{5-8}$ In this context, we recently reported the highly diastereoselective synthesis of tetralin-fused spirooxindoles with two contiguous (quaternary/tertiary) stereogenic centers via hydride shift triggered $\mathrm{C}\left(\mathrm{sp}^{3}\right)-\mathrm{H}$ bond functionalization (internal redox reaction, upper part of Scheme 1)..$^{9-15}$ Application of the 
construction of related structure with two contiguous quaternary stereogenic centers is not a trivial issue from the following two viewpoints: (1) difficulty of the stereoselective construction of fused-structure with two contiguous quaternary stereogenic centers, and (2) their potent biological activity.

Herein we report the realization of the target reaction (lower part of Scheme 1). Interestingly, the replacement of nitrogen atom to oxygen atom in the target structure with two contiguous quaternary stereogenic centers could be accomplished under the optimized reaction conditions, and corresponding adducts were obtained in good chemical yields with excellent diastereoselectivities (up to $d . r .=>20: 1$ ). This transformation realized net oxygen atom induced hydride shift/cyclization process, which is sometimes difficult in the substrate with low reactive electrophilic portion.

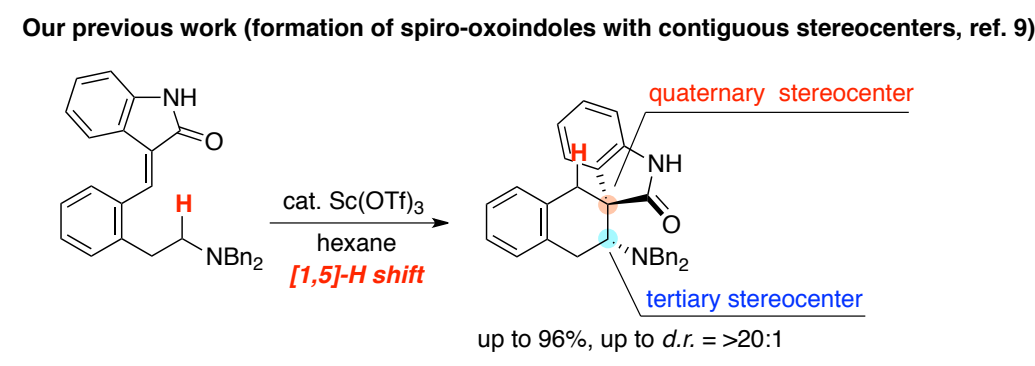

This work (formation of spiro-oxoindoles with contiguous quaternary stereocenters)

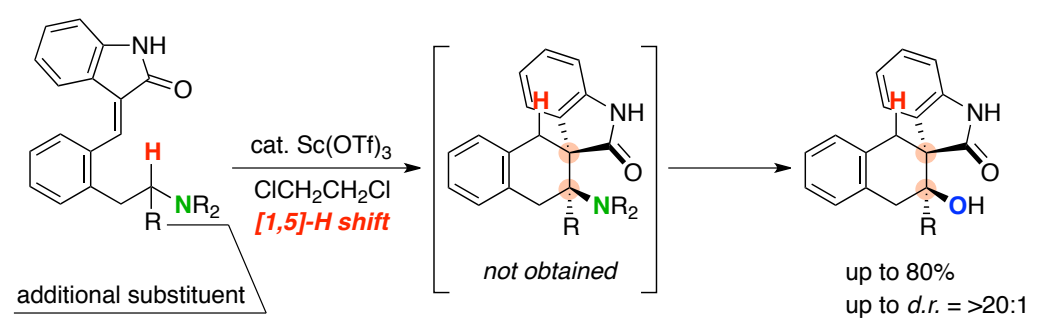

Scheme 1. Stereoselective synthesis of tetralin-fused spirooxindoles with two contiguous quoternaty stereoters.

First trial was conducted with 1a having methyl-group adjacent to nitrogen atom (Table 1). When 1a was treated with $10 \mathrm{~mol} \%$ of $\mathrm{Sc}(\mathrm{OTf})_{3}$ in $\mathrm{ClCH}_{2} \mathrm{CH}_{2} \mathrm{Cl}$ at $50{ }^{\circ} \mathrm{C}$, starting material 1a was mostly consumed after $12 \mathrm{~h}$. But surprisingly, the resulting product was not the desired adduct $\mathbf{2 a}$, but tertiary alcohol $\mathbf{3 a}$, whose structure was determined by X-ray analysis (57\%, entry 1). ${ }^{16}$ This unexpected result is quite impressive for us. One of the limitation of the internal redox reaction, especially using benzylidene oxindole as a 
substrate, ${ }^{9,12}$ is the requirement of nitrogen atom; that is, high electron donating ability of nitrogen was essential to promote the key hydride shift process, and the reaction with corresponding oxygen analogue did not proceed completely. From this viewpoint, the present reaction realized the net oxygen atom induced hydride shift/cyclization process, and hence, would open the new door of the related reaction. Stimulated by this result, our attention turned to tertiary alcohol $\mathbf{3 a}$ as a target. Further studies suggested that $\mathrm{Sc}(\mathrm{OTf})_{3}$ was the catalyst of choice. Although $\mathrm{Yb}(\mathrm{OTf})_{3}$ and $\mathrm{Gd}(\mathrm{OTf})_{3}$ also promoted the reaction, the chemical yields reached only low level (entries 2 and 3). Same situation hold for other catalysts such as $\mathrm{Mg}(\mathrm{OTf})_{2}, \mathrm{Zn}(\mathrm{OTf})_{2}, \mathrm{Al}(\mathrm{OTf})_{3}$, and $\mathrm{BF}_{3} \bullet \mathrm{OEt}_{2}$ (entries 4-7).

Solvent screening offered promising information for improving the chemical yield. Although the starting material $\mathbf{1 a}$ was mostly consumed in $\mathrm{CH}_{2} \mathrm{Cl}_{2}$ as in the case of $\mathrm{ClCH}_{2} \mathrm{CH}_{2} \mathrm{Cl}$, amine product $\mathbf{2 a}$ was solely observed (ca. 70\%, entry 8). This result was specific in $\mathrm{CH}_{2} \mathrm{Cl}_{2}$, i.e., the reaction in $\mathrm{CH}_{3} \mathrm{CN}$ resulted in exclusive formation of 3a (58\%, entry 9), and the reaction did not proceed when $\mathrm{MeOH}$ and Benzene were employed (entries 10 and 11). Synthesis of alcohol 3a was also possible even in the $\mathrm{CH}_{2} \mathrm{Cl}_{2}$ by the additional heating for $3 \mathrm{~h}$ in the presence of $\mathrm{H}_{2} \mathrm{O}$ (solvent amount) after the formation of amine 2a. Gratifyingly, the chemical yield of 3a was significantly improved to $80 \%$ (entry 12). Scale-up reaction was also attainable in this sequential system in $\mathrm{CH}_{2} \mathrm{Cl}_{2}$, and satisfactory chemical yield (71\%) was achieved (entry 13$)$.

Table 1. Optimization of reaction conditions. ${ }^{a}$
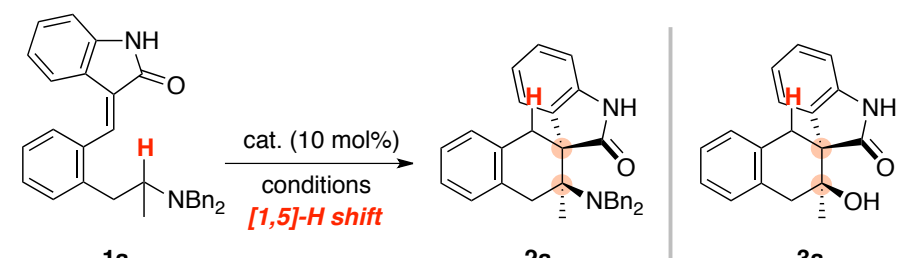

$1 \mathrm{a}$

2a

\begin{tabular}{|c|c|c|c|c|}
\hline \multirow{2}{*}{ Entry } & \multirow{2}{*}{ Catalyst } & \multirow{2}{*}{ Solvent } & \multicolumn{2}{|c|}{ Yield/ \% ${ }^{b}$} \\
\hline & & & $\mathbf{3 a}$ & $2 \mathbf{a}^{\mathrm{c}}$ \\
\hline 1 & $\mathrm{Sc}(\mathrm{OTf})_{3}$ & $\mathrm{ClCH}_{2} \mathrm{CH}_{2} \mathrm{Cl}$ & $52(>20: 1)$ & 0 \\
\hline 2 & $\mathrm{Yb}(\mathrm{OTf})_{3}$ & $\mathrm{ClCH}_{2} \mathrm{CH}_{2} \mathrm{Cl}$ & $4(>20: 1)$ & $0(80)$ \\
\hline 3 & $\mathrm{Gd}(\mathrm{OTf})_{3}$ & $\mathrm{ClCH}_{2} \mathrm{CH}_{2} \mathrm{Cl}$ & $16(>20: 1)$ & $0(65)$ \\
\hline
\end{tabular}




\begin{tabular}{ccccc}
4 & $\mathrm{Mg}(\mathrm{OTf})_{2}$ & $\mathrm{ClCH}_{2} \mathrm{CH}_{2} \mathrm{Cl}$ & $26(>20: 1)$ & $0(74)$ \\
5 & $\mathrm{Zn}(\mathrm{OTf})_{2}$ & $\mathrm{ClCH}_{2} \mathrm{CH}_{2} \mathrm{Cl}$ & trace & $0(98)$ \\
6 & $\mathrm{Al}(\mathrm{OTf})_{3}$ & $\mathrm{ClCH}_{2} \mathrm{CH}_{2} \mathrm{Cl}$ & $18(>20: 1)$ & $0(63)$ \\
7 & $\mathrm{BF}_{3} \bullet \mathrm{OEt}_{2}$ & $\mathrm{ClCH}_{2} \mathrm{CH}_{2} \mathrm{Cl}$ & $5(>20: 1)$ & $0(73)$ \\
8 & $\mathrm{Sc}_{(\mathrm{OTf})_{3}}$ & $\mathrm{CH}_{2} \mathrm{Cl}_{2}$ & 0 & ca. $70(0)$ \\
9 & $\mathrm{Sc}(\mathrm{OTf})_{3}$ & $\mathrm{CH}_{3} \mathrm{CN}$ & $58(>20: 1)$ & $0(30)$ \\
10 & $\mathrm{Sc}(\mathrm{OTf})_{3}$ & $\mathrm{MeOH}$ & 0 & $0(98)$ \\
11 & $\mathrm{Sc}(\mathrm{OTf})_{3}$ & $\mathrm{Benzene}$ & $8(>20: 1)$ & $0(62)$ \\
$12^{\mathrm{d}}$ & $\mathrm{Sc}(\mathrm{OTf})_{3}$ & $\mathrm{CH}_{2} \mathrm{Cl}_{2}$ & $80(>20: 1)$ & $0(0)$ \\
$13^{\text {e }}$ & $\mathrm{Sc}(\mathrm{OTf})_{3}$ & $\mathrm{CH}_{2} \mathrm{Cl}_{2}$ & $72(>20: 1)$ & $0(0)$ \\
\hline
\end{tabular}

${ }^{a}$ Unless otherwise noted, all reactions were conducted with $0.10 \mathrm{mmol}$ of $1 \mathrm{a}$ in the presence of an acid catalyst $(10 \mathrm{~mol} \%)$ in solvent $(1.0 \mathrm{~mL})$ at $50{ }^{\circ} \mathrm{C} .{ }^{\mathrm{b}}$ Isolated yield. ${ }^{\mathrm{c}}$ Recovery of $1 \mathrm{a}$ was shown in parenthesis. ${ }^{\mathrm{d}} \mathrm{H}_{2} \mathrm{O}(1.0 \mathrm{~mL}$, solvent amount $)$ was added after $12 \mathrm{~h}$, then heated for $3 \mathrm{~h}$ at refluxing temperature. ${ }^{\mathrm{e}} 2.53 \mathrm{mmol}$ scale.

Figure 1 shows the plausible mechanism of the present reaction. At first, $[1,5]-\mathrm{H}$ shift/cyclization process proceeds to give $\mathrm{NBn}_{2}$-substituted compound 2a. Importantly, because of the high electron donating ability of nitrogen atom, there is ring opening/closing equilibrium between 2a and A. After above events, two pathways could be assumed for the formation of 3a: (1) hydrolysis of iminium species $\mathbf{A}$ to ketone moiety $\mathbf{B}$ followed by the intramolecular aldol reaction (path I), and (2) acid induced liberation of $\mathrm{NBn}_{2}$ group followed by the nucleophilic addition of $\mathrm{H}_{2} \mathrm{O}$ to the resulting tertiary carbocation $\mathbf{D}$ (path II). By considering the chelation model $\mathbf{C}$ and hydrogen bonded transition state structure $\mathbf{E}$, stereochemical outcome could be well rationalized in both cases. As for the high stereoselectivity, thermodynamic control after the hydrolysis (interconversion between 3a and 3a') was another possible phenomena.

To gain insight into the reaction mechanism, some additional experiments were conducted (Figure 2). When $\mathrm{NBn}_{2}$-substituted spirocompound 2a was treated with 10 equiv. of $\mathrm{NaBH}_{4}$ in the presence of $10 \mathrm{~mol} \%$ of $\mathrm{Sc}(\mathrm{OTf})_{3}$, tertiary amine 4 and secondary alcohol 5 were detected (60\% combined yield), and alkane-type product 6 was not obtained at all. This result strongly suggests that path I would be a main pathway. Subjection of 
the each diastereomers $3 \mathbf{c}$ and 3c' to the reaction conditions (10 mol\% of $\mathrm{Sc}(\mathrm{OTf})_{3}$, $\mathrm{ClCH}_{2} \mathrm{CH}_{2} \mathrm{Cl}, 50{ }^{\circ} \mathrm{C}$ ) resulted in no production of another diastereomer, which implies the stereochemistry is determined in kinetically.

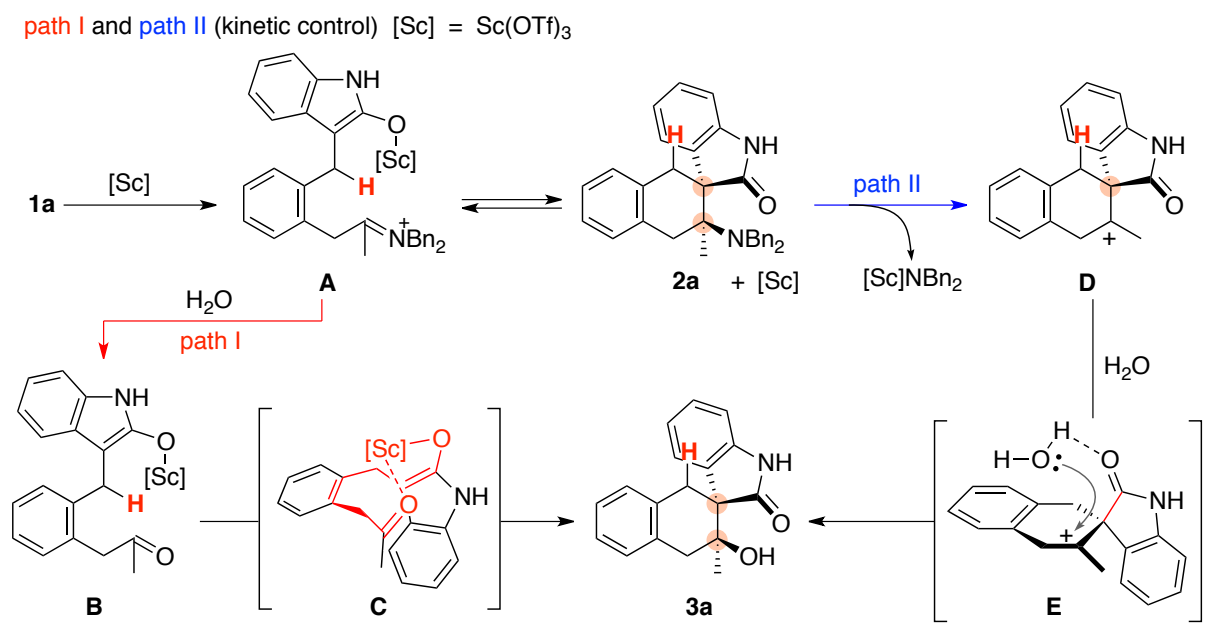

path III (thermodynamic control)

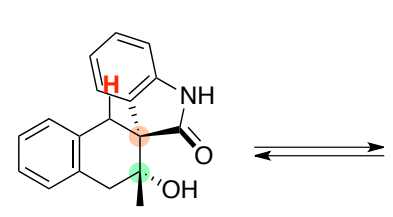

$3 a^{\prime}$<smiles>CCCCCC(c1ccccc1CC=O)c1c(O)[nH]c2ccccc12</smiles>

B

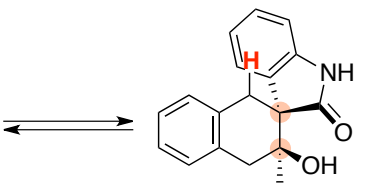

$3 a$

Figure 1. Possible reaction mechanisms.

Reaction in the presence of $\mathrm{NaBH}_{4}$ (reducing reagent)

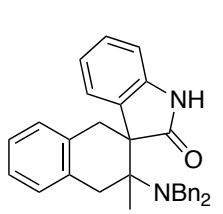

$2 a$

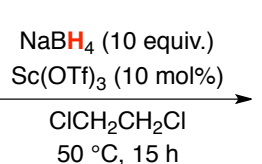

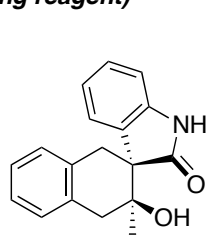

$24 \%$

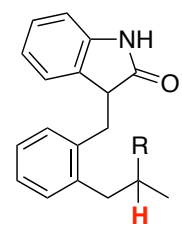

$60 \%$ (the ratio of $4 / 5, N D$ ) 6 : not observed $4\left(\mathrm{R}=\mathrm{NBn}_{2}\right) \mathbf{5}(\mathrm{R}=\mathrm{OH})$

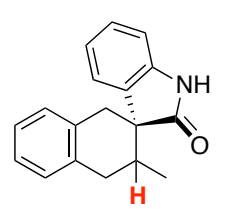

Checking of acid mediated equilibrium between 3a and 3a'.

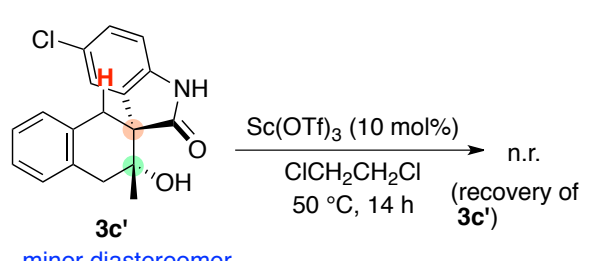

minor diastereomer

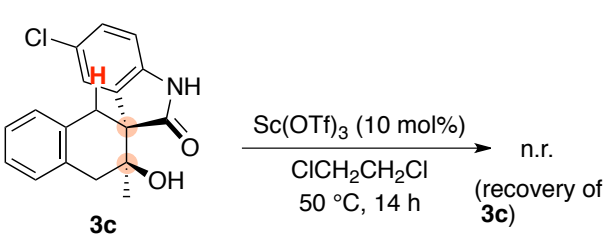

major diastereomer

n.r. $=$ no reaction

Figure 2. Additional experiments for clarification of the mechanism. 
Having the optimal reaction conditions and reaction mechanism, substrate scope was examined (Figure 3). This reaction was applicable to various substituted-oxindole moieties, and corresponding adducts $\mathbf{3 a - c}$ were obtained in good chemical yields with good to excellent diastereoselectivities (up to $80 \%, d . r .=>20: 1$ ). Further studies revealed that the substituent effect on mother aromatic ring was almost negligible. Tetralin-fused spirooxindoles 3d-h having electron donating groups (methyl and methoxy) and electron withdrawing group (fluoro), and naphthyl-type product $3 \mathbf{i}$ were obtained good chemical yields and diastereoselectivities $(45-69 \%, d . r .=7.7->20: 1)$.
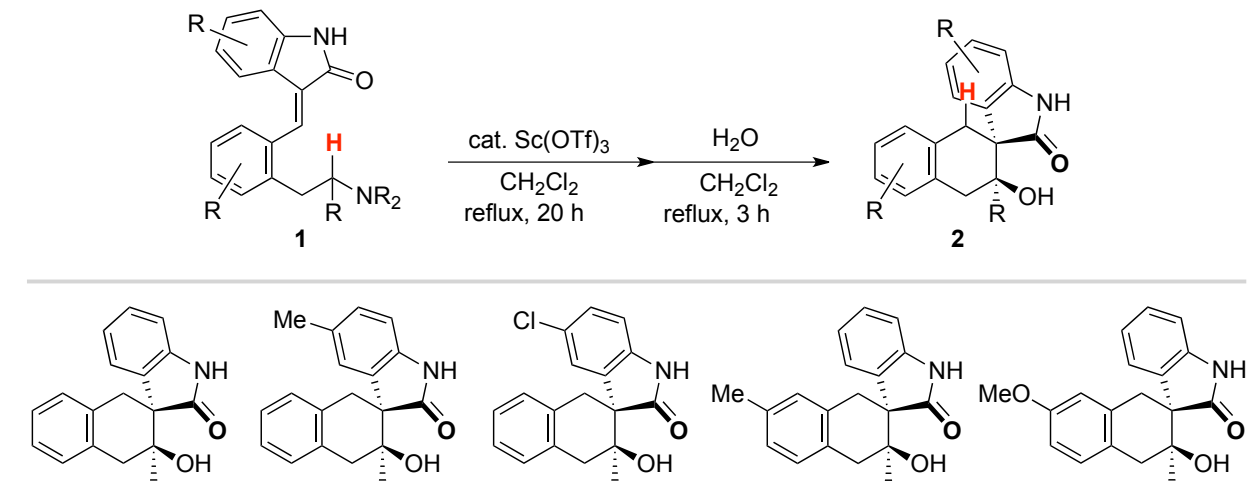

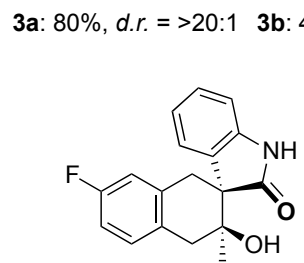

3f: $69 \%$, d.r. $=7.7: 1$

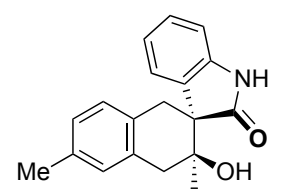

$3 \mathbf{g}: 63 \%$, d.r. $=14: 1$

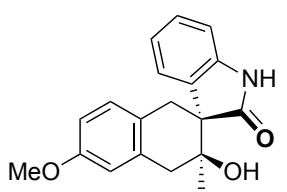

3h: $46 \%$, d.r. $=10: 1$

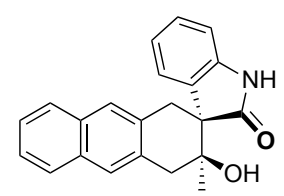

3i: $45 \%$, d.r. $=>20: 1$

Figure 3. Substrate cope.

In summary, we developed the highly diastereoselective synthesis of tetralin-fused spirooxindoles with two contiguous quaternary stereogenic centers via hydride shift triggered $\mathrm{C}\left(\mathrm{sp}^{3}\right)-\mathrm{H}$ bond functionalization. The unique feature of the present reaction is the replacement of nitrogen atom to oxygen atom. Some additional experiments revealed that this replacement occurred in the meanwhile ring-opening/closing equilibrium. Furthers studies of this "atom-replacement" strategy for the construction of another hybrid structure is under way in our laboratory.

\section{Acknowledgments}


This work was partially supported by a Grant-in-Aid for Scientific Research from the Japan Society for the Promotion of Science, and by grants from The Naito Foundation. 


\section{References and notes}

1. Some reviews on hybrid-type natural products, see: (a) K. C. Nicolaou, C. R. H. Hale, C. Nilewski, H. A. Loannidou, Chem. Soc. Rev. 2012, 41, 5185; (b) K. Kitamura, Y. Ando, T. Matsumoto, K. Suzuki, Chem. Rev. 2018, 118, 1495; (c) K. Ma, B. S. Martin, X. Yin, M. Dai, Nat. Prod. Rep. 2019, 36, 174; (d) L. A. Morrill, R. B. Susick, J. V. Chari, N. K. Garg, J. Am. Chem. Soc. 2019, 141, 12423; (e) Y.-J. Hu, L.-X. Li, J. C. Han, L. Min, C.-C. Li, Chem. Rev. 2020, 120, 5910.

2. For recent reviews on the synthesis of spirooxindoles, see: (a) C. V. Galliford, K. A. Scheidt, Angew. Chem. Int. Ed., 2007, 46, 8748; (b) B. M. Trost, M. K. Brennan, Synthesis 2009, 3003; (c) F. Zhou, Y.-L. Liu, J. Zhou, Adv. Synth. Catal. 2010, 352, 1381. d) N. R. Ball-Jones, J. J. Badillo A. K. Franz, Org. Biomol. Chem. 2012, 10, 5165; (e) L. Hong, R. Wang, Adv. Synth. Catal. 2013, 355, 1023; (f) D. Cheng, Y. Ishihara, B. Tan, C. F. Barbas III, ACS Catal. 2014, 4, 743.

3. (a) M. Rottmann, C. McNamara, B. K. S. Yeung, M. C. S. Lee, B. Zou, B. Russell, P. Seitz, D. M. Plouffe, N. V. Dharia, J. Tan, S. B. Cohen, K. R. Spencer, G. E. González-Páez, S. B. Lakshminarayana, A. Goh, R. Suwanarusk, T. Jegla, E. K. Schmitt, H. P. Beck, R. Brun, F. Nosten, L. Renia, V. Dartois, T. H. Keller, D. A. Fidock, E. A. Winzeler, T. T. Diagana, Science, 2010, 329, 1175; (b) S. Y. Leong, P. W. Smith, B. Zou, Chin. J. Chem. 2014, 32, 1217; (c) S. Wang, W. Sun, Y. Zhao, D. McEachern, I. Meaux, C. Barrière, J. A. Stuckey, J. L. Meagher, L. Bai, L. Liu, C. G. Hoffman-Luca, J. Lu, S. Shangary, S. Yu, D. Bernard, A. Aguilar, O. Dos-Santos, L. Besret, S. Guerif, P. Pannier, D. Gorge-Bernat, L. Debussche, Cancer Res. 2014, 74, 5855.

4. For some natural products and pharmaceuticals containing tetralin scaffold, see: (a) O. Pinto, J. Sardinha, P. D. Vaz, F. Piedade, M. J. Calhorda, R. Abramovitch, N. Nazareth, M. Pinto, M. S. J. Nascimento, A. P. Rauter, J. Med. Chem. 2011, 54, 3175; (b) M. Kvasnica, L. Rarova, J. Oklestkova, M. Budesinsky L. Kohout, Bioorg. Med. Chem. 2012, 20, 6969; (c) J. H. Soper, S. Sugiyama, K. Herbst-Robinson, M. J. James, X. Wang, J. Q. Trojanowski, A. B. Smith III, V. M. Y. Lee, C. Ballatore, K. R. Brunden, ACS Chem. Neurosci. 2012, 3, 928; (d) M. J. Hatfield, L. G. Tsurkan, J. L. Hyatt, C. C. 
Edwards, A. Lemoff, C. Jeffries, B. Yan, P. M. Potter, J. Nat. Prod. 2013, 76, 36; (e) Gamal-Eldeen, M.; Hamdy, N. A.; Abdel-Aziz, H. A.; El-Hussieny, E. A.; Fakhr, I. M. I. Eur. J. Med. Chem. 2014, 77, 323.

5. F. Manoni, S. J. Connon, Angew. Chem. Int. Ed. 2014, 53, 2628.

6. B. Wang, H.-J. Leng, X.-Y. Yang, B. Han, C.-L. Rao, L. Liu, C. Peng, W. Huang, RSC. $A d v . \mathbf{2 0 1 5}, 8,88272$.

7. H.-R. Wu, L. Cheng, D.-L. Kong, H.-Y. Huang, C.-L. Gu, L. Liu, D. Li, Wang, C.-J.. Org. Lett. 2016, 18, 1382.

8. K. Lokesh, V. Kesavan, Eur. J. Org. Chem. 2017, 5689.

9. M. Machida, K. Mori, Chem. Lett. 2018, 47, 868

10. For the internal redox reaction developed by our group, see: (a) K. Mori, Y. Ohshima, K. Ehara, T. Akiyama, Chem. Lett. 2009, 38, 524; (b) K. Mori, T. Kawasaki, S. Sueoka, T. Akiyama, Org. Lett. 2010, 12, 1732; (c) K. Mori, S. Sueoka, T. Akiyama, J. Am. Chem. Soc. 2011, 133, 2424; (d) K. Mori, S. Sueoka, T. Akiyama, Chem. Lett. 2011, 40, 1386; (e) K. Mori, K. Ehara, K. Kurihara, T. Akiyama, J. Am. Chem. Soc. 2011, 133, 6166; (f) K. Mori, T. Kawasaki, T. Akiyama, Org. Lett. 2012, 14, 1436; (g) K. Mori, K. Kurihara, T. Akiyama, Chem. Commun. 2014, 50, 3729; (h) K. Mori, N. Umehara, T. Akiyama, Adv. Synth. Catal. 2015, 357, 901; (i) T. Yoshida, K. Mori, Chem. Commun. 2017, 53, 4319; (j) K. Yokoo, K. Mori, Chem. Commun. 2018, 54, 6927; (k) N. Hisano, Y. Kamei, Y. Kansaku, M. Yamanaka, K. Mori, Org. Lett. 2018, 20, 4223; (1) T. Yoshida, K. Mori, K. Chem. Commun. 2018, 54, 12686; (m) R. Tamura, E. Kitamura, R. Tsutsumi, M. Yamanaka, T. Akiyama, K. Mori, Org. Lett. 2019, 21, 2383; (n) Y. Otawa, K. Mori, Chem. Commun. 2019, 55, 13856; (o) K. Yokoo, K. Mori, Org. Lett. 2020, 22, 244; (p) D. Hoshino, K. Mori, Org. Bioomol. Chem. 2020, 18, 6602.

11. For the double $\mathrm{C}\left(\mathrm{sp}^{3}\right)-\mathrm{H}$ bond functionalization by sequential utilization of the internal redox reaction developed by our group, see: (a) K. Mori, K. Kurihara, S. Yabe, M. Yamanaka, T. Akiyama, J. Am. Chem. Soc. 2014, 136, 3744; (b) K. Mori, N. Umehara, T. Akiyama, Chem. Sci. 2018, 9, 7327; (c) K. Mori, R. Isogai, Y. Kamei, M. Yamanaka, T. Akiyama, J. Am. Chem. Soc. 2018, 140, 6203; (d) M. Kataoka, Y. Otawa, N. Ido, K. Mori, Org. Lett. 2019, 21, 9334. (e) K. Yokoo, D. Sakai, K. Mori, Org. Lett. 2020, 22, 
5801.

12. For the synthesis of tetrahydroquinoline fused-spirooxindoles by internal redox reaction, see: a) Y.-Y. Han, W.-Y. Han, X. Hou, X.-M. Zhang, W.-C. Yuan. Org. Lett. 2012, 14, 4054. Asymmetric version, see: b) W. Cao, X. Liu, J. Guo, L. Lin, X. Feng, Eur. Chem. J. 2015, 21, 1632.

13. For recent reviews on internal redox process, see: (a) M. Tobisu, N. Chatani, Angew. Chem. Int. Ed. 2006, 45, 168; (b) S. C. Pan, Beilstein J. Org. Chem. 2012, 8, 1374; (c) M. Wang, ChemCatChem 2013, 5, 1291. d) B. Peng, N. Maulide, Chem, Eur. J. 2013, 19, 13274; (e) L. Wang, J. Xiao, Adv. Synth. Catal. 2014, 356, 1137; (f) M. C. Haibach, D. Seidel, Angew. Chem. Int. Ed. 2014, 53, 5010; (g) S. J. Kwon, D. Y. Kim, Chem. Rec. 2016, 16, 1191; (h) X.-D. An, J. Xiao, Org. Chem. Front. 2021, 8, 1364.

14. For selected recent examples of the internal redox reactions, see: (a) S. J. Pastine, K. M. McQuaid, D. Sames, J. Am. Chem. Soc. 2005, 127, 12180; (b) S. J. Pastine, D. Sames, Org. Lett. 2005, 7, 5429; (c) C. Zhang, C. Kanta De, R. Mal, D. Seidel, J. Am. Chem. Soc. 2008, 130, 416; (d) K. M. McQuaid, D. Sames, J. Am. Chem. Soc. 2009, 131, 402; (e) K. M. McQuaid, J. Z. Long, D. Sames, Org. Lett. 2009, 11, 2972; (f) S. Yang, Z. Li, X. Jian, C. He, Angew. Chem. Int. Ed. 2009, 48, 3999; (g) P. A. Vadola, D. Sames, J. Am. Chem. Soc. 2009, 131, 16525; (h) I. D. Jurberg, B. Peng, E. Wöstefeld, M. Wasserloos, N. Maulide, Angew. Chem. Int. Ed. 2012, 51, 1950; (i) D.-F. Chen, Z.-Y. Han, Y.-P. He, J. Yu, L.-Z. Gong, Angew. Chem. Int. Ed. 2012, 51, 12307; (j) Y.-Z. Li, M.-L.; Zhao, W.-F. Chang, X. Wen, H. Sun, Q.-L. Xu, J. Org. Chem. 2015, 80, 9620; (k) K. Eamakumar, T. Maji, J. J. Partridge, J. A. Tunge, Org. Lett. 2017, 19, 4014; (1) S.-S. Li, L. Zhou, L. Wang, H. Zhao, L. Yu, J. Xiao, Org. Lett. 2018, 20, 138; (m) S. Wang, X. D. An, S. S. Li, X. Liu, Q. Liu, J. Xiao, Chem. Commun. 2018, 54, 13833; (n) S.-S. Li, S. Zhu, C. Chen, K. Duan, Q. Liu, J. Xiao, Org. Lett. 2019, 21, 1058; (o) S. Zhao, X. Wang, P. Wang, G. Wang, W. Xhao, X. Tang, M. Guo, Org. Lett. 2019, 21, 3990; (p) A. Paul, D. Seidel, J. Am. Chem. Soc. 2019, 141, 8788; (q) L. Zhou, Y.-B. Shen, X.-D. An, X.-J. Li, S.-S. Li, Q. Liu, J. Xiao, Org. Lett. 2019, 21, 8543; (r) L. Zhou, X.-D. An, S. Yang, X.-J. Li, C.-L. Shao, Q. Liu, J. Xiao, Org. Lett. 2020, 22, 776; (s) K. Duan, X.-D. An, L.-F. Li, L.-L. Sun, B. Qiu, X.-J. Li, C.-L. Shao, Q. Liu, J. 
Xiao, Org. Lett. 2020, 22, 2537.

15. For examples of the enantioselective internal redox reactions, see: (a) S. Murarka, I. Deb, C. Zhang, D. Seidel, J. Am. Chem. Soc. 2009, 131, 13226; (b) Y. K. Kang, S. M. Kim, D. Y. Kim, J. Am. Chem. Soc. 2010, 132, 11847; (c) W. Cao, X. Liu, W. Wang, L. Lin, X. Feng, Org. Lett. 2011, 13, 600; (d) G. Zhou, F. Liu, J. Zhang, Chem. Eur. J. 2011, 17, 3101; (e) Y.-P. He, Y.-L. Du, S.-W. Luo, L. Z. Gong, Tetrahedron Lett. 2011, 52, 7064; (f) L. Chen, L. Zhang, Z. Lv, J.-P. Cheng, S. Luo, Chem. Eur. J. 2012, 18, 8891; (g) Z.-W. Jiao, S.-Y. Zhang, C. He, Y.-Q. Tu, S.-H. Wang, F.-M. Zhang, Y.-Q. Zhang, H. Li, Angew. Chem. Int. Ed. 2012, 51, 8811; (h) Y. K. Kang, D. Y. Kim, Adv. Synth. Catal. 2013, 355, 3131; (i) Y. K. Kang, D. Y. Kim, Chem. Commun. 2014, 50, 222; (j) C. W. Suh, D. Y. Kim, Org. Lett. 2014, 16, 5374; (k) W. Cao, X. Liu, J. Guo, L. Lin, X. Feng, Chem. Eur. J. 2015, 21, 1632. See also, refs 10e and 11c.

16. CCDC-2081088 contains the supplementary crystallographic data of 3a (See SI for details). This data can be obtained free of charge from The Cambridge Crystallographic Data Center via www.ccdc.cam.ac.uk/data_request. 